\section{Avaliação da qualidade da informação do Sistema de Informação Perinatal (SIP-CLAP /OPAS) para monitoramento da assistência perinatal hospitalar, Belo Horizonte, 2004}

\author{
Assessment of the quality of information \\ from the Perinatal Information System (SIP- \\ CLAP/OPAS) used to monitor hospital \\ perinatal care, Belo Horizonte, 2004
}

Maria Albertina Santiago Rego 1

Elisabeth Barboza França 2

Deise Campos Cardoso Afonso 3
1 Departamento de Pediatria. Faculdade de Medicina. Universidade Federal de Minas Gerais. Av. Alfredo Balena, 190. Belo Horizonte, MG, Brasil. CEP: 30.130-100.

E-mail: albertin@medicina.ufmg.br.

2 Programa de Pós-Graduação em Saúde da Criança e do Adolescente. Departamento de Pediatria. Faculdade de Medicina. Universidade Federal de Minas Gerais. Belo Horizonte, MG, Brasil.

3 Programa de Pós-Graduação em Saúde Pública. Departamento de Medicina Preventiva e Social. Faculdade de Medicina. Universidade Federal de Minas Gerais. Belo Horizonte, MG, Brasil.

\section{Resumo}

Objetivo: avaliar a qualidade da informação do SIP-CLAP/OPAS em maternidades de Belo Horizonte.

Métodos: para avaliação da completude foi analisada amostra aleatória sistemática de 562 formulários SIP e prontuários médicos correspondentes, coletados durante a assistência em duas maternidades, em 2004. Uma subamostra de $20 \%$ foi utilizada para avaliação da confiabilidade do banco de dados eletrônico do programa. Foram calculadas proporções de completude antes e após resgate da informação disponível em prontuário, e o indice Kappa e o coeficiente de correlação intraclasse (ICC), com nível de significância de 5\%, para análise da concordância.

Resultados: a completude de variáveis do SIP durante a assistência foi em média $72 \%$ no Hospital 1 e $86 \%$ no Hospital 2. O ganho médio percentual após busca da informação em prontuários foi de $18 \%$ e $7 \%$, respectivamente. Foram observados indices muito bons de concordância nos dois hospitais.

Conclusões: o SIP-CLAP representa uma alternativa para monitoramento da assistência hospitalar perinatal, mas apresenta problemas para sua utilização adequada, pois a completude avaliada durante a assistência não foi satisfatória. São necessários investimentos para o aprimoramento do programa nos hospitais, fundamentais para obtenção de indicadores essenciais para a qualificação da assistência hospitalar ao parto e nascimento.

Palavras-chave Avaliação de serviços de saúde, Sistemas de informação, Assistência perinatal, Mortalidade infantil 


\section{Introdução}

No Brasil, os problemas perinatais constituem a principal causa de óbito em crianças menores de cinco anos. Grande parcela desses óbitos pode ser reduzida com a melhoria da qualidade da assistência perinatal, por meio da regionalização, integração da assistência e implementação de intervenções relativamente simples e de alta efetividade, nos níveis ambulatorial e hospitalar, durante a gestação, parto, nascimento e período neonatal. ${ }^{1}$ Sabe-se que um dos principais objetivos do acompanhamento ao prénatal, parto e puerpério é a identificação de fatores que possam colocar a mãe e o recém-nascido sob maior risco de resultados adversos, em um momento que se possa intervir para evitar ou reduzir esses resultados, 2 embora haja certo consenso que a identificação dos problemas e o conhecimento das melhores intervenções disponíveis não são suficientes para garantir a melhoria da qualidade do cuidado. 3

Um componente fundamental na qualificação da assistência individual e no monitoramento do cuidado perinatal no nível coletivo é a existência de um sistema de informação4-6 que contenha indicadores utilizados durante o processo assistencial, fazendo da coleta e do registro dos dados uma prática sistemática dos serviços de saúde.7-9

No Brasil, os sistemas de informação de rotina que permitem avaliar o período perinatal -Sistema de Informações sobre Nascidos Vivos (SINASC) e Sistema de Informações sobre Mortalidade (SIM) são instrumentos importantes para a vigilância do nascimento e óbito. Entretanto, a informação disponível nesses sistemas não contempla adequadamente o processo assistencial ao parto e período neonatal.1,9,10 A utilização de informação disponível em prontuários hospitalares convencionais para o monitoramento do cuidado e produção do conhecimento também se torna limitada, devido, principalmente, à dificuldade na definição e adoção de critérios padronizados para o registro clínico, o que pode alterar substancialmente os resultados dos estudos comparativos. 11-13

Na América Latina, o Sistema de Informação Perinatal do Centro Latino Americano de Perinatologia e Desenvolvimento Humano da Organização PanAmericana da Saúde (SIP-CLAP/OPAS) foi concebido em resposta às necessidades dos países da região, com altos índices de mortalidade materna e perinatal não compatíveis com o grau de desenvolvimento socioeconômico. ${ }^{14}$ A proposta incorpora o conceito da utilização da informação em rede para a melhoria da qualidade da assistência15,16 e vem sendo utilizada em mais de 20 países da região, concomitante ao uso dos sistemas formais de registros dos eventos vitais de nascimento e óbito, principalmente no Uruguai e Argentina.14,17 Diferentemente da Rede Neonatal VermontOxford, 18 a maior rede internacional de monitoramento da assistência de recém-nascidos de muito baixo peso ao nascer, o modelo do SIP-CLAP propõe o registro uniformizado da informação perinatal durante o processo assistencial para todos os nascimentos, integrando as estratégias de melhoria da assistência individual às funções de vigilância da saúde perinatal no nível coletivo. A rede brasileira de pesquisas neonatais que utiliza protocolos nos moldes da Rede Vermont-Oxford congrega alguns serviços de referência em atenção perinatal e vem desenvolvendo principalmente estudos de pesquisa clínica em recém-nascidos abaixo de $1500 \mathrm{~g}$ de peso ao nascer. 19

A elaboração dos formulários clínicos do SIPCLAP foi precedida pela análise das normas existentes, com consensos sobre os dados mínimos indispensáveis para a atenção perinatal. O formulário básico de registro da informação, a história clínica perinatal (HCP-SIP), é complementado por outros que são utilizados somente em situações específicas. Dessa forma se obtém um instrumento modular cuja complexidade cresce para os casos de risco. A informação contida na HCP-SIP é considerada essencial e deve estar presente para todas as parturientes, puérperas e recém-nascidos. Durante a função assistencial ou após sua finalização, a própria equipe de saúde pode ingressar os dados no computador e em qualquer momento pode utilizar os programas do SIP para obter estatísticas da população assistida. Os vários módulos do programa são de fácil manejo e constituem uma ferramenta de autoavaliação e de investigação autônomas. A integração da atenção hospitalar com a atenção ambulatorial se faz por meio do cartão perinatal, concretizando o conceito de rede assistencial.16,20

A análise da informação gerada pelo sistema permite o monitoramento da qualidade da assistência no local onde ela é realizada e também na região onde está implementada. O registro e a análise da informação gerada no local do cuidado desencadeiam um processo institucional mais abrangente de compreensão da gestão do cuidado, e possibilitam o monitoramento dos eventos ao redor do nascimento. Além disso, tem a vantagem de ser um programa de domínio público, desenvolvido por órgão técnico da Organização Pan-Americana da Saúde (OPAS), com características adequadas às demandas de monitoramento da assistência perinatal 
para a melhoria da qualidade do cuidado. O SIPCLAP torna-se então um instrumento de monitoramento da assistência ao parto e nascimento, acompanhando variáveis chaves para prevenção dos óbitos materno, fetal e neonatal, possibilitando a implementação de normas gerenciais e assistenciais que promovam equidade e subsidiem intervenções apropriadas. $9,14,16,17,20$

No Brasil, apesar da tendência crescente de utilização de indicadores assistenciais para monitoramento do desempenho dos serviços de saúde, questões relativas à produção e gestão da informação, essenciais para garantir a existência e qualidade dos dados, são frequentemente ignoradas ou consideradas como de menor importância. Para que um sistema de informação possa ser utilizado com os objetivos para os quais ele foi desenvolvido é necessário que as informações registradas sejam completas e confiáveis. ${ }^{11}$ A completude, medida como proporção da presença de registros em relação ao total de variáveis a ser registrada, e a confiabilidade da informação em relação ao registro das variáveis em prontuários médicos convencionais são metodologias correntemente utilizadas em estudos brasileiros de avaliação da qualidade da informação do SINASC21,22 e do SIM.13 Por outro lado, em relação ao SIP-CLAP, não foram localizados estudos similares de avaliação da sua completude e confiabilidade, exceto do banco de dados eletrônico divulgados em manuais do programa.17 Ressalta-se que, no caso deste sistema, a completude da informação regis-trada no formulário da HCP-SIP durante o processo assistencial, e que posteriormente alimenta o banco de dados eletrônico do programa, pode ser consi-derada um parâmetro indicativo da incorporação do programa na assistência hospitalar.23

Desta forma, este trabalho propõe avaliar a completude da informação registrada no formulário HCP-SIP durante o processo assistencial hospitalar perinatal e a confiabilidade do banco de dados eletrônico do sistema para monitoramento da assistência hospitalar em Belo Horizonte.

\section{Métodos}

Estudo de completude e confiabilidade da informação de variáveis selecionadas do SIP-CLAP, em duas maternidades de referência em assistência perinatal hospitalar de Belo Horizonte, por meio do grau de preenchimento da HCP-SIP durante a assistência, e da concordância da informação arquivada no banco de dados eletrônico do programa, comparada à informação registrada no prontuário da mãe e do recémnascido, segundo dois componentes:
Componente A: avaliação da qualidade da informação do SIP-CLAP na assistência hospitalar perinatal, através da análise de completude da HCP-SIP durante a assistência, antes e após busca da informação em prontuário;

Componente B: avaliação da qualidade da informação do SIP-CLAP para monitoramento da assistência perinatal hospitalar, através da análise de concordância da informação eletrônica do programa (SIP-eletrônico) comparada à informação registrada em prontuário.

Três hospitais/maternidades, responsáveis pela assistência de mais da metade do volume obstétrico hospitalar do Sistema Único de Saúde (SUS) em Belo Horizonte tinham o SIP-CLAP implantado em 2004. Foram então selecionados dois destes hospitais com diferentes modelos assistenciais, conforme descrito a seguir.

O Hospital 1, universitário federal, prioriza programas de ensino e pesquisa nos níveis da graduação e pós-graduação vinculados à assistência em áreas da saúde e afins, e faz parte da rede hospitalar de referência estadual para gestações de alto risco perinatal. Em 2004, foram realizados cerca de 3000 partos. A HCP-SIP utilizada era fotocopiada em preto e branco a partir da original em amarelo nas "caselas" que indicam risco. A versão utilizada do SIP-CLAP era 1.5, no sistema operacional DOS.

O Hospital 2 é uma fundação filantrópica de referência para a gestão SUS em programas de humanização da atenção perinatal implementados em nível nacional. Utilizava a versão 1.2 no sistema operacional Windows, que era a última versão disponibilizada em 2004 pelo Centro Latino Americano de Perinatologia, com ampliação da base de dados. Foram realizados cerca de 6000 partos no ano.

Para o estudo de completude, calculou-se um tamanho de amostra de 280 partos para cada hospital, utilizando-se os seguintes parâmetros: frequência esperada de completude de $50 \%$, precisão de $6 \%$, nível de confiança de $95 \%$ e perdas estimadas de $15 \%$. Utilizou-se amostra aleatória sistemática dos partos ocorridos nos dois hospitais em 2004, com seleção sequencial segundo ordem prédefinida e sorteio do primeiro valor para garantia do critério de aleatoriedade. Para o estudo de confiabilidade foi utilizada subamostra aleatória sistemática de $20 \%$ do total de prontuários analisados no estudo de completude em cada uma das maternidades, correspondendo a 56 registros clínicos em cada hospital. Porém, no Hospital 2 sete registros não foram localizados (três não constavam no banco de dados SIP-CLAP e quatro prontuários não foram 
encontrados). Optou-se por não realizar reposição dessas perdas $(\mathrm{n}=7)$ devido a problemas operacionais e por se considerar que a amostra analisada era representativa. O período de coleta de dados estendeu-se de abril a junho de 2004 no Hospital 1 e de novembro de 2004 a janeiro de 2005 no Hospital 2.

Tendo em vista a utilização de dois modelos de história clinica perinatal (versões diferentes da HCPSIP e dos programas eletrônicos do SIP-CLAP) pelos dois hospitais estudados, a coleta de dados contemplou somente variáveis presentes nas duas versões utilizadas, sendo realizada em duas etapas, de acordo com os componentes do estudo:

\section{Componente A: análise de completude}

Para esse componente do estudo, foram utilizadas duas fontes de dados: o formulário HCPSIP e o prontuário hospitalar convencional.

Para cada dupla "mãe-recém-nascido" foi realizada transcrição das informações registradas na HCP-SIP preenchida pelos profissionais durante o processo assistencial em um novo documento, denominado formulário-pesquisa. Os dados eram colhidos em visitas diárias aos hospitais após o fechamento do prontuário pela equipe assistencial, mas anteriormente ao processo de transferência da informação para o meio eletrônico. O objetivo desse procedimento foi avaliar se estavam disponíveis durante a assistência à mãe e ao recém-nascido conforme o fluxo hospitalar do SIP-CLAP: a) a HCP-SIP é preenchida pelos profissionais durante o processo assistencial à mãe e ao recém-nascido, desde a internação até a alta hospitalar e, b) após a alta, os dados são digitados e arquivados no banco de dados eletrônico do programa, com revisão manual para correção de dados incompletos e análise de consistência eletrônica, para consolidação final dos dados.

Em seguida, foi realizada busca no prontuário das informações faltantes na HCP-SIP para completar o formulário-pesquisa. Assim, foi possível verificar quais informações estavam ausentes durante o processo assistencial na HCP-SIP e se tais informações estavam registradas no prontuário.

Foi considerada ausência da informação na HCP-SIP quando não havia registro da informação nos campos correspondentes de acordo com a estrutura do formulário. Entretanto, no prontuário, para as variáveis "patologias maternas" e "patologias neonatais", a não menção de patologia(s) na evolução clínica ou outros locais do prontuário foi considerada como ausência de patologia, de acordo com o modelo de registro da informação clínica em prontuários convencionais.

As variáveis selecionadas foram classificadas em cinco módulos, como na HCP-SIP: a) Sóciodemográficas; b) História obstétrica pregressa; c) Gestação atual; d) Parto e nascimento; e) Recémnascido.

Os procedimentos foram executados por bolsistas capacitados, selecionados através de currículo, interesse por pesquisa e conhecimento em informática, e foram supervisionados pela pesquisadora principal.

\section{Componente B: análise de confiabilidade}

Nesse componente do estudo, foram comparados os dados arquivados nos bancos de dados eletrônicos do SIP-CLAP, cedidos pelos hospitais participantes da pesquisa, com aqueles obtidos dos prontuários convencionais, resgatados dos respectivos serviços de arquivo médico hospitalar. Para possibilitar mascaramento, primeiramente foi realizada a coleta dos dados do prontuário em um novo formuláriopesquisa nos moldes do SIP-CLAP e só após foram solicitados aos hospitais os registros do banco de dados eletrônico do SIP-CLAP dos mesmos pacientes.

Foram consideradas todas as fontes de informação disponíveis nos prontuários, incluindo os exames complementares e documentos anexados (cartão perinatal, declaração de nascido vivo - DN), sendo priorizada, quando duplicada, a informação registrada durante a evolução clínica. Quando era verificada duplicidade e/ou inconsistência do valor da variável na evolução clínica, as informações consideradas eram as registradas no resumo de alta.

Esse componente foi executado pela pesquisadora principal devido à dependência de critérios técnicos na coleta da informação.

Nesta etapa foram selecionadas somente as variáveis que alcançaram maiores índices de completude em cada um dos módulos da história clínica perinatal: idade materna, número de gestações anteriores, fator Rh, idade gestacional estimada pelo obstetra, terminação do parto, peso ao nascer e Apgar de $1^{\circ}$ e $5^{\circ}$ minutos. As variáveis, motivo da indicação da cesariana, idade gestacional estimada pelo pediatra, condição do recém-nascido à alta e alimento do recém-nascido à alta foram acrescentadas, devido à relevância no processo assistencial e nos resultados perinatais obtidos.

As variáveis patologias maternas e patologias neonatais, embora determinantes dos resultados perinatais, foram excluídas da análise por exigirem inter- 
pretação técnica da informação registrada na evolução clínica, muitas vezes descrita sem o detalhamento e a clareza necessários.

Todas as variáveis estudadas eram comuns aos formulários utilizados nos dois hospitais.

A completude foi expressa como a proporção de HCP-SIP com registro da informação para as variáveis selecionadas, antes e após busca da informação em prontuário. Para cada variável comparouse a completude da HCP-SIP e da HCP-SIP combinada ao prontuário através do teste de $\mathrm{McNemar}$ para comparação de proporções dependentes, com nível de significância de 5\%.22 O índice de completude foi considerado como "bom" quando a presença da informação era maior que $90 \%$, "regular" quando esta proporção situava-se entre $70 \%$ e $90 \%$ e "precária" quando era inferior a 70\%.10

Para avaliar a confiabilidade, as informações do prontuário e do banco de dados eletrônico do SIPCLAP foram emparelhadas. No Hospital 1, três registros corresponderam a recém-nascidos que evoluíram para o óbito e, portanto, não foram incluídos na análise de concordância da variável "alimento à alta". Utilizou-se a estatística Kappa para medir a concordância das variáveis categóricas com intervalo de confiança de 95\%. Considerou-se concordância excelente para valores de Kappa acima de 0,80 , boa para valores entre 0,61 e 0,80 , moderada para valores entre 0,41 e 0,60 e fraca para valores de 0,40 ou menores.24,25 Para medir a concordância entre as variáveis contínuas utilizou-se o Coeficiente de Correlação Intraclasse ou Intraclass Correlation Coefficient (ICC), com intervalo de confiança de $95 \%$. Os valores de ICC podem variar entre -1 e +1 e foi considerada melhor a concordância quanto maior o valor observado. Considerou-se um coeficiente de concordância de $75 \%$ como valor mínimo aceitável. 25,26

Para a análise dos dados foram utilizados os programas Excel 5.0, SPSS versão 13.0, e Stata versão 9.0, 2005. O projeto foi autorizado pelas maternidades envolvidas no estudo e aprovado pela Comissão de Ética em Pesquisa da Universidade Federal de Minas Gerais (COEP-UFMG) em 24/11/2004, conforme Parecer ETIC no 313/04.

\section{Resultados}

Foram selecionadas 562 histórias clínicas perinatais preenchidas durante a assistência e os prontuários correspondentes para a análise de completude nos dois hospitais, e para a análise de confiabilidade, 105 histórias clínicas perinatais disponíveis em meio eletrônico e prontuários correspondentes.
Componente A: análise de completude da HCPSIP durante a assistência

A completude da informação sofreu oscilações de acordo com a variável e o hospital, conforme apresentado na Tabela 1. No Hospital 1, a completude média das variáveis registradas na HCP-SIP foi de $72 \%$ e de $90 \%$ quando somada à informação registrada em prontuário. Avaliando cada grupamento de variáveis separadamente, os seguintes percentuais médios foram registrados antes e após busca da informação em prontuário: variáveis sóciodemográficas: $81 \%$ e $87 \%$; história reprodutiva pregressa: $75 \%$ e $78 \%$; gravidez atual: $66 \%$ e $79 \%$; parto e nascimento: $61 \%$ e $96 \%$; recém-nascido: $78 \%$ e $96 \%$. O registro da informação aumentou significativamente para a maioria das variáveis (90\%) após coleta dos dados no prontuário.

No Hospital 2, a completude média das variáveis registradas na HCP-SIP foi de $86 \%$. Quando somada à informação disponível em prontuário, a completude média alcançou 92\%; semelhante, portanto, ao Hospital 1. A completude média das variáveis segundo os agrupamentos apresentou os seguintes resultados antes e depois da busca em prontuário: variáveis sócio-demográficas: 93\% e 96\%; história reprodutiva pregressa: $80 \%$ e $81 \%$; gravidez atual: $81 \%$ e $82 \%$; parto e nascimento: $85 \%$ e $98 \%$; recémnascido: $88 \%$ e $96 \%$. Após consulta em prontuário, o registro da informação aumentou significativamente para $57 \%$ das variáveis. Essa proporção, apesar de alta, foi menor que a observada no Hospital 1 . Observa-se que, globalmente, o Hospital 2 apresenta índices de completude da HCP-SIP mais altos quando comparados com o Hospital 1, com exceção das variáveis altura materna e patologias do recémnascido.

As menores completudes da HCP-SIP foram observadas para a variável patologias maternas no Hospital 1, e patologias do recém-nascido no Hospital 2, mas obtiveram recuperação quase total das informações após busca em prontuários nas duas instituições. Verificou-se ausência de informação em mais de 50\% das HCP-SIP no Hospital 1 para a indicação da cesariana, e no Hospital 2 para patologias maternas, também recuperadas após busca em prontuário.

Para algumas variáveis, principalmente as relativas à história reprodutiva pregressa e aos dados de pré-natal, a completude não alcançou índices altos, permanecendo, em alguns casos, com índices próximos ou abaixo de 70\%, mesmo após busca da informação em prontuário: mês e ano do término da última gestação, peso materno prévio à gravidez 
Tabela 1

Completude (\%) de variáveis perinatais registradas no SIP-CLAP/OPAS durante a assistência e após revisão do prontuário hospitalar, Belo Horizonte, 2004.

\begin{tabular}{|c|c|c|c|c|c|c|}
\hline \multirow{5}{*}{ Variáveis } & \multicolumn{3}{|c|}{ Hospital 1} & \multicolumn{3}{|c|}{ Hospital 2} \\
\hline & \multicolumn{3}{|c|}{$\%(N=277)$} & \multicolumn{3}{|c|}{$\%(N=285)$} \\
\hline & \multirow{3}{*}{$\begin{array}{l}\text { Completude } \\
\text { SIP }\end{array}$} & \multirow{3}{*}{\multicolumn{2}{|c|}{$\begin{array}{l}\text { Completude } \\
\text { SIP e } \\
\text { Prontuário }\end{array}$}} & \multirow{3}{*}{$\begin{array}{l}\text { Completude } \\
\text { SIP }\end{array}$} & \multicolumn{2}{|c|}{ Completude } \\
\hline & & & & & SIP e & $p^{*}$ \\
\hline & & & & & Prontuá & \\
\hline \multicolumn{7}{|l|}{ Condição sócio-demográfica } \\
\hline Idade materna & 89,2 & 100,0 & $<0,001$ & 91,9 & 98,2 & $<0,001$ \\
\hline Escolaridade materna & 75,5 & 75,8 & 1,000 & 93,7 & 94,4 & 0,500 \\
\hline Estado civil & 79,4 & 86,6 & $<0,001$ & 94,0 & 95,1 & 0,250 \\
\hline \multicolumn{7}{|l|}{ História obstétrica pregressa } \\
\hline Número de gestações anteriores & 93,9 & 100,0 & $<0,001$ & 95,8 & 98,2 & 0,016 \\
\hline Mês do término da última gestação ** & 65,6 & 66,7 & 0,250 & 72,0 & 72,0 & 1,000 \\
\hline Ano do término da última gestação ** & * 65,6 & 67,2 & 0,125 & 72,6 & 72,6 & 1,000 \\
\hline \multicolumn{7}{|l|}{ Gravidez atual } \\
\hline Número de consultas de pré-natal & 74,7 & 94,9 & $<0,001$ & 95,1 & 95,8 & 0,500 \\
\hline Peso materno antes da gestação atual & al 63,9 & 63,9 & 1,000 & 73,3 & 73,3 & 1,000 \\
\hline Altura materna & 54,9 & 54,9 & 1,000 & 52,6 & 52,6 & 1,000 \\
\hline Fator Rh & 82,7 & 99,3 & $<0,001$ & 98,2 & 98,9 & 0,500 \\
\hline VDRL & 60,6 & 98,9 & $<0,001$ & 90,5 & 92,6 & 0,031 \\
\hline Dúvidas em relação à IG & 57,8 & 61,7 & 0,001 & 77,2 & 78,6 & 0,125 \\
\hline \multicolumn{7}{|l|}{ Parto e nascimento } \\
\hline Patologias maternas & 22,7 & 100,0 & $<0,001$ & 41,8 & 97,9 & $<0,001$ \\
\hline IG estimada pelo obstetra & 76,9 & 99,3 & $<0,001$ & 95,4 & 98,6 & 0,004 \\
\hline Membranas antes do parto & 71,1 & 92,8 & $<0,001$ & 95,8 & 98,6 & 0,008 \\
\hline Início do parto & 66,1 & 91,3 & $<0,001$ & 88,8 & 95,8 & $<0,001$ \\
\hline Apresentação fetal & 71,5 & 92,4 & $<0,001$ & 90,5 & 93,7 & 0,004 \\
\hline Motivo da indicação da cesariana ${ }^{\dagger}$ & 45,6 & 98,0 & $<0,001$ & 84,6 & 100,0 & $<0,001$ \\
\hline Terminação do parto & 76,5 & 100,0 & $<0,001$ & 96,8 & 100,0 & 0,004 \\
\hline \multicolumn{7}{|l|}{ Recém-nascido } \\
\hline Peso ao nascer & 88,4 & 99,6 & $<0,001$ & 94,7 & 98,2 & 0,002 \\
\hline IG estimada pelo pediatra ${ }^{\dagger}$ & 56,4 & 62,4 & $<0,001$ & 90,4 & 94,0 & 0,002 \\
\hline Relação PN / IG †† & 80,4 & 94,1 & $<0,001$ & 86,9 & 95,8 & $<0,001$ \\
\hline Apgar $11^{+\dagger}$ & 90,4 & 100,0 & $<0,001$ & 99,2 & 99,2 & 1,000 \\
\hline Apgar $5^{\prime}+\dagger$ & 90,4 & 100,0 & $<0,001$ & 99,2 & 99,2 & 1,000 \\
\hline Patologias & 63,5 & 99,6 & $<0,001$ & 39,4 & 99,3 & $<0,001$ \\
\hline Condição do RN à alta †† & 75,6 & 100,0 & $<0,001$ & 91,1 & 98,9 & $<0,001$ \\
\hline Alimento do RN à alta $F$ & 69,2 & 96,6 & $<0,001$ & 89,5 & 97,5 & $<0,001$ \\
\hline Alojamento conjunto ${ }^{\dagger \dagger}$ & 78,6 & 100,0 & $<0,001$ & 91,0 & 91,8 & 1,000 \\
\hline Dia da alta ${ }^{\dagger \dagger}$ & 81,9 & 99,6 & $<0,001$ & 91,9 & 91,9 & 1,000 \\
\hline Mês da alta ${ }^{\dagger}$ & 81,9 & 99,6 & $<0,001$ & 91,9 & 91,9 & 1,000 \\
\hline
\end{tabular}

SIP-CLAP/OPAS = Sistema de Informação Perinatal do Centro Latino Americano de Perinatologia e Desenvolvimento Humano da Organização Pan-Americana da Saúde; VDRL = Venereal Disease Research Laboratory; PN = peso ao nascer; IG = idade gestacional; * = Teste de McNemar; ** = excluídas as primíparas; $\dagger$ = excluídos os partos vaginais; $\dagger \dagger=$ excluídos nascidos mortos; $F=$ excluídos nascidos mortos e óbitos neonatais. 
Completude (\%) de variáveis perinatais registradas no SIP-CLAP/OPAS durante a assistência e após revisão do prontuário hospitalar, Belo Horizonte, 2004.

\begin{tabular}{|c|c|c|c|c|c|c|}
\hline \multirow{2}{*}{ Variáveis } & \multicolumn{3}{|c|}{ Hospital 1} & \multicolumn{3}{|c|}{ Hospital 2} \\
\hline & $\mathrm{n}$ & $\mathrm{C}$ & IC95\% & $\mathrm{n}$ & C & IC95\% \\
\hline \multicolumn{7}{|l|}{ Concordância avaliada pelo ICC } \\
\hline Idade materna & 56 & 0,99 & $0,99-1,00$ & 49 & 0,99 & $0,98-0,99$ \\
\hline Número de gestações anteriores & 56 & 0,96 & $0,93-0,98$ & 49 & 0,90 & $0,83-0,94$ \\
\hline IG estimada pelo obstetra & 56 & 0,91 & $0,85-0,95$ & 49 & 0,99 & $0,98-0,99$ \\
\hline IG estimada pelo pediatra $\dagger$ & 52 & 0,97 & $0,95-0,98$ & 8 & 0,98 & $0,92-1,00$ \\
\hline Apgar $1^{\prime}+$ & 56 & 0,92 & $0,87-0,95$ & 49 & 1,00 & $1,00-1,00$ \\
\hline Apgar $5^{\prime}+$ & 56 & 0,99 & $0,99-1,00$ & 49 & 1,00 & $1,00-1,00$ \\
\hline Peso ao nascer & 56 & 1,00 & $1,00-1,00$ & 49 & 0,97 & $0,95-0,98$ \\
\hline \multicolumn{7}{|l|}{ Concordância avaliada pelo Kappa } \\
\hline Fator $\mathrm{Rh}$ & 44 & 1,00 & $0,70-1,00$ & 45 & 0,73 & $0,45-1,00$ \\
\hline Motivo da indicação da cesariana * & 20 & 0,46 & $0,29-0,64$ & 11 & 0,39 & $0,21-0,58$ \\
\hline Terminação do parto & 56 & 1,00 & $0,77-1,00$ & 49 & 0,86 & $0,63-1,00$ \\
\hline Alimento do recém-nascido à alta ** & 40 & 0,62 & $0,39-0,84$ & 44 & - & - \\
\hline Condição do recém-nascido à alta † & 56 & 0,87 & $0,67-1,00$ & 49 & 0,68 & $0,48-0,88$ \\
\hline
\end{tabular}

SIP-CLAP = Sistema de Informação Perinatal do Centro Latino Americano de Perinatologia; ICC = coeficiente de correlação intraclasse; IC95\% = Intervalo de $95 \%$ de confiança; C = proporção observada de concordância entre o SIPeletrônico e o prontuário; IG = idade gestacional; ${ }^{*}=$ excluídos partos vaginais; $\dagger=$ excluídos nascidos mortos; ${ }^{*}=$ excluídos os nascidos mortos e óbitos neonatais.

atual e altura materna em ambos os hospitais. $\mathrm{O}$ mesmo foi observado para as variáveis dúvidas em relação à idade gestacional e idade gestacional estimada pelo pediatra no Hospital 1.

A completude da HCP-SIP no Hospital 1 foi em torno de $70 \%$ ou menos para algumas variáveis relacionadas ao parto e ao recém-nascido, mas alcançaram índices acima de $90 \%$ após busca em prontuário: número de consultas de pré-natal, VDRL, membranas antes do parto, início do parto, apresentação fetal, indicação do parto operatório, condição do recém-nascido à alta, alimento à alta. Para o Hospital 2 observou-se aumento importante na completude de algumas variáveis que, entretanto, já apresentavam completude acima de $80 \%$ na HCPSIP, com destaque para início do parto, indicação do parto operatório e relação peso ao nascer e idade gestacional.

Componente B: análise de confiabilidade da informação eletrônica do SIP-CLAP

A Tabela 2 apresenta a análise de confiabilidade utilizando ICC, para as variáveis contínuas, e Índice Kappa para as variáveis categóricas. Observa-se que o número de pares analisados (n) variou de acordo com a disponibilidade da informação nas duas fontes de dados no total de 56 pares no Hospital 1 e 49 no Hospital 2.

Encontrou-se concordância acima de 0,80 entre as informações registradas no arquivo eletrônico do SIP-CLAP e as disponíveis em prontuário, para a maioria das variáveis (83\%) analisadas no Hospital 1. No Hospital 2, este índice foi verificado para uma proporção menor de variáveis $(73 \%)$. Os menores percentuais de concordância foram encontrados para a variável motivo da indicação da cesariana, 0,46 no Hospital 1 e 0,39 no Hospital 2. Verificou-se que a variável fator $\mathrm{Rh}$, com índice de concordância de 1,00 no Hospital 1, obteve proporção de somente 0,73 no Hospital 2. A variável idade gestacional estimada pelo pediatra, com concordância global acima de 0,90 , foi avaliada em somente oito registros no Hospital 2 para os quais foi possível realizar o pareamento. $\mathrm{O}$ registro da variável alimento à alta no Hospital 2, com o total dos pares $(n=44)$ com 
registro de leite materno em uma das fontes dos dados impossibilitou a análise de confiabilidade utilizando a estatística Kappa.

\section{Discussão}

No presente estudo, a completude da informação da HCP-SIP registrada durante o processo assistencial foi avaliada como "regular a boa" em dois hospitais de referência em assistência perinatal em Belo Horizonte em 2004. Verificou-se que, no Hospital 2, o registro da informação está em grande parte no formulário da HCP-SIP, com cerca de dois terços das variáveis com índice de completude considerado "bom" e pouca modificação dos índices na maioria das variáveis analisadas após busca da informação complementar em prontuário, sugerindo utilização do instrumento durante o processo assistencial. $\mathrm{O}$ Hospital 1, por outro lado, apresentou índices de completude menos satisfatórios, indicando que a informação para a assistência baseia-se principalmente nos registros de prontuário. A utilização, neste hospital, de HCP-SIP fotocopiadas em preto e branco por ocasião do estudo pode ter contribuído para esses resultados. O documento alterado em sua concepção pode, inclusive, ter perdido características básicas importantes para a assistência individual, pois a HCP-SIP ressalta as variáveis com resultados desfavoráveis indicadores de risco, quando as respostas são registradas nas "caselas" amarelas, com indicação para a tomada de decisões clínicas que, por sua vez, serão respaldadas pelo registro sequenciado da informação nos vários momentos da assistência.16,20 Outro fator a ser ressaltado foi a não utilização, nas duas instituições, dos formulários complementares para os casos de alto risco perinatal.

Alguns outros aspectos do registro da informação pelos profissionais da assistência podem ser avaliados no contexto do cuidado perinatal, segundo o período do processo reprodutivo e mesmo na análise individual da disponibilidade da variável e sua completude. A ausência de informação relativa ao peso anterior à gestação atual, altura materna e intervalo interpartal em proporção importante de HCP-SIP nos dois hospitais, e que não foram recuperados após busca em prontuário, podem indicar que essas variáveis, coletadas durante o pré-natal, são pouco utilizadas durante a assistência hospitalar ao parto e nascimento.

Baixo preenchimento de variáveis não relacionadas diretamente com o processo assistencial à mãe e ao recém-nascido foi encontrado também por Simini et al., ${ }^{23}$ em estudo de avaliação da implemen- tação do SIP-CLAP em maternidades da América Latina e Caribe. Conde-Agudelo e Belizán, ${ }^{27}$ utilizando como fonte de dados para estudos epidemiológicos o SIP-CLAP de instituições de países da América Latina, correspondentes aos anos 1985-97, encontraram proporções elevadas de ausência de registro de variáveis demográficas maternas e da gestação. Ausência quase total do registro de peso e altura materna nos prontuários médicos foi encontrada em estudo realizado no município do Rio de Janeiro entre julho de 1999 e fevereiro de 2001, nos estratos 1 (hospitais municipais e federais) e 2 (hospitais privados conveniados com o SUS, militares, estaduais e filantrópicos). ${ }^{12}$ Também o número de consultas de pré-natal foi preenchida somente em cerca de metade dos prontuários avaliados no Rio de Janeiro, 12,21 enquanto no SIP-CLAP essa variável teve maior completude (75\% no Hospital 1 e 95\% no Hospital 2).

Algumas variáveis, como intervalo interpartal, não foram analisadas nos estudos de qualidade avaliados, provavelmente por não serem registradas rotineiramente nos prontuários médicos, sendo uma característica importante da HCP-SIP a possibilidade de registro dessa e outras variáveis associadas ao prognóstico perinatal. Dessa forma, a fragmentação dos dados em prontuários, consequente à coleta não sistematizada da informação e não padronização de terminologia, dificulta a utilização adequada da documentação clínica tanto para melhoria da qualidade da assistência quanto para estudos epidemiológicos7,12,28,29 e pode levar ao sub-registro de alguns indicadores maternos não utilizados diretamente na assistência ao parto e nascimento, mas que são reconhecidamente importantes na abordagem da saúde materna e neonatal, nos resultados perinatais e na definição de plano de cuidados após a alta hospitalar. Variáveis relacionadas à história reprodutiva materna, como o baixo peso ao nascer em gestações prévias, são contempladas na HCP-SIP e com frequência não são registradas em prontuários convencionais.

A completude superior a $80 \%$ da relação peso ao nascer para idade gestacional no Hospital 1, e o registro de somente $56 \%$ no SIP e $62 \%$ após busca no prontuário para a variável idade gestacional estimada pelo pediatra durante a assistência, sugerem que a idade gestacional estimada pelo obstetra está sendo utilizada para uma parcela importante de recém-nascidos para a qual não se dispõe de aferição da idade gestacional pelo pediatra. Esse fato é aceitável quando a idade gestacional estimada pelo obstetra é definida por ultrassom precoce e/ou data confiável da última menstruação. 30 Essas aferições, 
que definem condutas clínicas para o recém-nascido, precisam ser adequadamente documentadas durante o processo assistencial.

De toda forma, a melhoria do registro da informação após busca em prontuário verificada nesse estudo não deve ser interpretada como excelência na qualidade da informação dos prontuários convencionais. O aumento importante nas proporções de completude relativas às patologias maternas $\mathrm{e}$ neonatais nos dois hospitais após busca da informação nos prontuários pode estar refletindo, pelo menos parcialmente, a metodologia aqui utilizada. Isso porque o não registro de patologia no prontuário foi considerado como informação presente (no caso, ausência de doença), enquanto no formulário do SIPCLAP era necessário preencher a casela correspondente a não doença. Também para outras variáveis, e principalmente para o Hospital 2, a utilização de documentos arquivados no prontuário (cópias xerográficas do cartão perinatal, resultados de exames, declaração de nascido vivo - DN) mesmo sem o registro da informação na evolução clínica diária, pode ter elevado o índice de completude encontrado nos prontuários.

A qualidade da informação eletrônica, medida pelo índice Kappa e ICC, mostrou-se adequada, com concordância excelente para a maioria das variáveis nos dois hospitais. Entretanto, destaca-se o encontro de baixos índices de concordância para algumas variáveis relevantes do processo assistencial e definidoras de aspectos importantes da saúde materna e neonatal. Entre esses, destaca-se a verificação de concordância moderada a fraca para a variável motivo da indicação da cesariana. Mudanças de procedimentos e suas indicações durante a evolução materna e processo assistencial podem explicar parcialmente esses resultados, mas não pode ser excluída a possibilidade de certo grau de distanciamento entre o registro da informação e as ações implementadas na assistência individual à mãe. $\mathrm{O}$ índice de concordância de 0,62 , com intervalo de confiança de $95 \%$ muito amplo para a variável alimento do recém-nascido à alta em um dos hospi- tais, pode estar relacionado à presença de informação ambígua no prontuário, registrada possivelmente por diferentes categorias profissionais em evoluções dos médicos e enfermeiras. No Hospital 2, a ausência de variabilidade no registro dessa variável na HCP-SIP impossibilitou a análise estatística de concordância da variável. É necessário reavaliar estes resultados em estudo delineado especificamente para essas variáveis. Erros na entrada de dados no SIP eletrônico podem também ser responsáveis por parte dos resultados encontrados, como no caso da variável fator Rh, onde o índice de concordância no Hospital 2 pode ser considerado baixo.

Em síntese, nesse estudo procurou-se avaliar tanto a confiabilidade das variáveis disponíveis no banco de dados eletrônico do SIP quanto sua possível utilização durante o processo assistencial, em dois hospitais com modelos assistenciais complementares. Verificou-se alta concordância para a maioria das variáveis estudadas no SIP-eletrônico, mas a completude da HCP-SIP preenchida durante a assistência à mãe e ao recém-nascido não foi satisfatória, principalmente em um dos hospitais. Considerando as características do SIP-CLAP, programa de domínio público de registro uniformizado da informação básica do processo assistencial para todos os nascimentos, recomenda-se maiores investimentos na implantação do programa nos hospitais, de importância fundamental para obtenção de indicadores essenciais para a qualificação da assistência em serviços de atenção hospitalar ao nascimento. Outros estudos devem ser realizados para melhor compreender as questões envolvidas no registro e valorização da informação clínica.

\section{Agradecimentos}

As autoras agradecem o apoio financeiro da Fapemig - $n^{\circ}$ REF: EDT 1770/2003 e do CNPq - Processo ${ }^{\circ}$ 403707/2004-8, e a Ana Paula Travassos pelo apoio na análise estatística.

\section{Referências}

1. Victora CG. Intervenções para reduzir a mortalidade infantil e pré-escolar e maternal no Brasil. Rev Bras Epidemiol. 2001; 4:3-69.

2. American Academy of Pediatrics. The American College of Obstetrics and Gynecologists. Guidelines for perinatal care. $5^{\mathrm{a}}$ ed. Washington: AAP/ACOG; 2002.
3. Sinclair JC. Evidence-based therapy in neonatology: distilling the evidence and applying it in practice. Acta Paediatr. 2004; 93: 1146-52.

4. Knippenberg R, Lawn JE, Darmstadt GL, Begkoyian G, Fogstad H, Walelign N, Paul VK, Lancet Neonatal Survival Steering Team. Systematic scalling up of neonatal care in countries. Lancet. 2005; 365: 1087-98. 
5. Mann MY, Lloyd-Puryear AM, Linzer D. Enhancing communication in the 21st century. Pediatrics. 2006; 117 (Suppl 5): 315-9.

6. AbouZahr C, Boerma T. Health information systems: the foundations of public health. Bull World Health Organ. 2005; 83: 578-83.

7. Diaz-Rossello JL. Health services research, outcomes, and perinatal information systems. Curr Opin Pediatr. 1998; 10: 117-22.

8. Rodrigues RJ. Information systems: the key to evidencebased health practice. Bull World Health Organ. 2000; 78: 1344-51.

9. Parada CMGL, Barros FC. Análise comparativa entre o sistema informático perinatal desenvolvido pelo CLAP/OPS e os sistemas de informações brasileiros. Rev Paul Pediatr. 2001; 19: 170-6.

10. Almeida MF, Alencar GP, Novaes HMD, Ortiz LP. Sistemas de informação e mortalidade perinatal: conceitos e condições de uso em estudos epidemiológicos. Rev Bras Epidemiol. 2006; 9: 56-68.

11. Schout D, Novaes HMD. Do registro ao indicador: gestão da produção da informação assistencial nos hospitais. Ciênc Saúde Coletiva. 2007; 12: 935-44.

12. Campos MR, Leal MC, Souza Jr PR, Cunha CB. Consistência entre fontes de dados e confiabilidade interobservador do estudo da morbi-mortalidade e atenção perinatal e neonatal no município do Rio de Janeiro. Cad Saúde Pública. 2004; 20 (Supl 1): 34-43.

13. Pedrosa LD C O, Sarinho SW, Ordonha MR. Análise da qualidade da informação sobre causa básica de óbitos neonatais registrados no Sistema de Informações sobre Mortalidade: um estudo para Maceió, Alagoas, Brasil, 2001-2002. Cad Saúde Pública. 2007; 23: 2385-95.

14. Simini F. Perinatal information system (SIP): a clinical data base in Latin American and the Caribbean. Lancet. 1999; 354(9172): 75 .

15. Molin J. A regional perinatal database in southern Sweden - a basis for quality assurance in obstetrics and neonatology. Acta Obstet Gynecol Scand. 1997; 76: 37-9.

16. Simini F, Rubino M, Lopez R, Gonzalo-Diaz A, Schwarcz R. Processamento de datos del sistema informático perinatal. Montevidéo: CLAP; 1996.

17. Simini F. Sistema informático perinatal en el Uruguay 15 años de datos. 1985-1999. Montevideo: CLAP; 2001.

18. Horbar JD, Plsek PE, Schriefer JA, Leahy K. Evidencebased quality improvement in neonatal and perinatal medicine: the neonatal intensive care quality improvement collaborative experience. Pediatrics. 2006; 118 (Suppl 2): 57-64.

Recebido em 10 de setembro de 2008

Versão final apresentada em 25 de março de 2009

Aprovado em 12 de junho de 2009
19. Barros FC, Diaz-Rossello JL. Redes multicêntricas e a qualidade da atenção perinatal. J Pediatr (Rio J). 2004; 80: 254-6.

20. Fescina RH, Butrón B, De Mucio B, Martínez G, DíazRossello JL, Camacho V, Simini F, Mainero L. Sistema informático perinatal: história clínica perinatal. Montevidéo: CLAP/SMR; 2007.

21. Theme-Filha MM, Gama SGN, Cunha CB, Leal MC. Confiabilidade do sistema de informações sobre nascidos vivos hospitalares no município do Rio de Janeiro, 1999. 2001. Cad Saúde Pública. 2004; 20 (Supl 1): 83-91.

22. Romero M, Dalia E, Cunha, C. Avaliação da qualidade das variáveis epidemiológicas e demográficas do Sistema de Informações sobre Nascidos Vivos, 2002. Cad Saúde Pública. 2007; 23: 701-14.

23. Simini F, Fernández A, Sosa C, Díaz-Rossello JD. Sistema informático perinatal: latencia de incorporación e impacto em el registro clínico perinatal. Ginecol Obstet Méx. 2001; 69: 386-9.

24. Altman DG. Practical Statistics for Medical Research. London, England: Chapman \& Hall; 1991.

25. Szklo M, Nieto FJ. Epidemiology: beyond the basics. Boston: Jones and Bartlett Publishers; 2004.

26. Shrout PE, Fleiss JL. Intraclass Correlations: Uses in Assessing Rater Reliability. Psycol Bull. 1979; 86: 420-8.

27. Conde-Agudelo A, Belizán JM. Maternal morbidity and mortality associated with interpregnancy interval: cross sectional study. BMJ. 2000; 321: 1255-9.

28. Reisch LM, Fosse JS, Beverly K, Yu O, Barlow WE, Harris EL, Rolnick S, Barton MB, Geiger AM, Herrinton LJ, Greene SM, Fletcher SW, Elmore JG. Trainig, quality assurance, and assessment of medical record abstraction in a multisite study. Am J Epidemiol. 2003; 157: 546-51.

29. Thomas EJ, Studdert DM, Brennan TA. The reliability of medical record review for estimating adverse event rates. Ann Inter Med. 2002; 136: 812-6.

30. Abuhamad AZ, ACOG Committee on Practice BulletinsObstetrics. ACOG Practice Bulletin: clinical management guidelines for obstetrician-gynecologists number 98 . Ultrasonography in pregnancy. Obstet Gynecol. 2008; 112: 951-61. 